RECEIVED ........; ACCEPTED .........

Preprint typeset using $\mathrm{LT}_{\mathrm{E}} \mathrm{X}$ style emulateapj v. 04/21/05

\title{
CAN OVERTURNING MOTIONS IN PENUMBRAL FILAMENTS BE DETECTED?
}

\author{
LOKESh Bharti ${ }^{1}$, MANFREd SCHÜSSLER ${ }^{1}$, AND MATTHIAS REMPEL ${ }^{2}$ \\ ${ }^{1}$ Max-Planck-Institut für Sonnensystemforschung, Max-Planck-Str. 2, 37191 Katlenburg-Lindau, Germany, and \\ ${ }^{2}$ High Altitude Observatory, NCAR, P.O. Box 3000, Boulder, CO 80307, USA \\ Received ......... Accepted ..........
}

\begin{abstract}
Numerical simulations indicate that the filamentation of sunspot penumbrae and the associated systematic outflow (the Evershed effect) are due to convectively driven fluid motions constrained by the inclined magnetic field. We investigate whether these motions, in particular the upflows in the bright filaments and the downflows at their edges can be reliably observed with existing instrumentation. We use a snapshot from a sunspot simulation to calculate 2D maps of synthetic line profiles for the spectral lines FeI $7090.4 \AA$ and CI $5380.34 \AA$. The maps are spatially and spectrally degraded according to typical instrument properties. Line-of-sight velocities are determined from line bisector shifts. We find that the detectability of the convective flows is strongly affected by spatial smearing, particularly so for the downflows. Furthermore, the line-of-sight velocities are dominated by the Evershed flow unless the observation is made very near to disk center. These problems may have compromised recent attempts to detect overturning penumbral convection. Lines with a low formation height are best suited to detect the convective flows.
\end{abstract}

Subject headings: Sun: convection - sunspots - Sun: magnetic field

\section{INTRODUCTION}

Significant progress in understanding the physical processes underlying the photospheric structure and dynamics of sunspots has been made in recent years by the interplay of observational results obtained with advanced instrumentation on the ground and in space with the results of comprehensive numerical simulations. In particular, simulations indicate that magneto-convective processes dominate the dynamics and structure formation in the umbra (Schüssler \& Vögler 2006, Bharti et al. 2010a) and penumbra (Heinemann et al. 2007, Scharmer et al. 2008, Rempel et al. 2009ab, Rempel 2011). Sunspot fine structure seen in the photosphere, such as umbral dots, light bridges, and penumbral filaments, are attributed to overturning convection in the presence of a vertical or inclined magnetic field.

Observational evidence has been found in support of the notion of magneto-convection in the umbra (Bharti et al. 2007ab, 2009, Ortiz et al. 2010) and in light bridges (Bharti et al. 2007c, Rimmele 2008, Rouppe van der Voort et al. 2010). Observational support for convection in penumbral filaments is not so clearcut, although some indirect evidence has been found by Márquez et al. (2006) and Sánchez Almeida et al. (2007). From numerical models there are two aspects to this flow that are manifest in observations: Upflows in bright filaments in the inner penumbra lead to dark cores and particularly bright filament heads moving toward the umbra during their formation phase (Rempel et al. 2009a); along the full length of filaments upflows are deflected by the Lorentz force due to the inclined magnetic field and turn into the almost horizontal Evershed flows along the filaments near the surface of optical depth unity (Rempel 2011). Part of the upflowing material, however, turns over in a roll-type motion perpendicular to the direction of the filament axis and descends in downflows along the edges of the filaments. Indirect evidence for this kind of overturning flows is provided by the observations of 'twisting' motions (Ichimoto et al. 2007, Bharti et al. $2010 b)$ in penumbral filaments. The associated upflow veloc-

Electronic address: bharti@mps.mpg.de ities seem to be high enough to balance the radiative losses of filaments over an extended part of their length (Zakharov et al. 2008, Bharti et al. 2010b). On the other hand, the associated downflows along the edges of penumbral filaments have not yet been clearly detected by spectroscopic observations (e.g., Franz \& Schlichenmaier 2009, Katsukawa \& Jurcak 2010, Louis et al. 2011). For instance, Bellot Rubio et al. (2010) used high-resolution observations of the line Fe I $7090.4 \AA$ line with the Swedish 1-m telescope (Scharmer et al. 2003) on La Palma and found no such downflows above their detection limit of a few hundred $\mathrm{m} \mathrm{s}^{-1}$. In fact, the vertical penumbral velocity field derived by these authors appears to be dominated by upflows along the line of sight.

The aim of this paper is to study, using the most advanced numerical simulations of sunspots, under which conditions overturning convective flows in penumbral filaments of the kind present in the simulations would be detectable in observations. Thereby, we wish to clarify whether negative results such as those of Bellot Rubio et al. (2010) are in fact in contradiction to those of the numerical simulations.

\section{SIMULATIONS, LINE SYNTHESIS AND CONVOLUTION}

We consider a single snapshot from a sunspot simulation using the MURaM code (for details, see Vögler et al. 2005, Rempel et al. 2009b) with a grid resolution of $16 \mathrm{~km}$ in vertical and $32 \mathrm{~km}$ in the horizontal directions. The dimensions of the computational box are $49.152 \times 49.152 \mathrm{Mm}^{2}$ horizontally and 6.144 Mm in depth. While this simulation shares the basic ingredients with Rempel et al. 2009b, the numerical setup consists of an individual sunspot (instead of the sunspot pair). To obtain extended penumbrae in presence of periodic boundaries (imposing same polarity spots nearby), we artificially increased the inclination angle at the top boundary $(700 \mathrm{~km}$ above the quiet sun photosphere) by increasing the horizontal field components by a factor of two compared to a potential field. The total (solar) time elapsed since initialization of the simulation is about $6 \mathrm{~h}$. The sunspot has a total flux of about $10^{22} \mathrm{Mx}$, the fraction of the spot area covered by penumbra (defined through $0.5<I / I_{\odot}<0.9$ ) is $65 \%$. The first 3.3 hours 
were run in a resolution of $24 \mathrm{~km}$ in vertical and $48 \mathrm{~km}$ in the horizontal directions, the following 2.7 hours were run in the high resolution of $16 \times 32 \mathrm{~km}^{2}$. The last 26 min were performed in addition with non-grey radiative transfer using 4 opacity bins optimized for the quiet-Sun conditions. This sunspot is part of a convergence study in which the influence of the grid resolution on penumbral fine structure is investigated (Rempel 2010, Rempel in prep.).

For the analysis carried out in this paper, we consider a $20.5 \times 20.5 \mathrm{Mm}^{2}(640 \times 640$ pixel horizontally $)$ section of the snapshot, covering a quarter of the simulated sunspot (cf. Figure 11). To determine synthetic line profiles we used the STOPRO (STOkes PROfiles) code (Berdyugina et al. 2003), which calculates the Stokes parameters for spectral lines in local thermodynamic equilibrium (LTE). We obtained intensity (Stokes- $I$ ) profiles for the two lines FeI $7090.4 \AA$ and CI $5380.34 \AA$ for each of the $640 \times 640$ pixels of the simulation snapshot. The first of these lines has been used in the study of Bellot Rubio et al. (2010), so that we can directly compare with their results. The line is particularly well suited for velocity measurements since its Landé factor is zero. The outer wings of this line are formed around $100 \mathrm{~km}$ above optical depth unity (Bellot Rubio et al. 2005). The CI 5380.34 $\AA$ line forms in the deep photosphere (Fleck 1991) and thus is more sensitive to the higher convective velocities in these layers.

The maps of synthetic intensity profiles were considered a) at original simulation resolution, and b) smeared and rebinned spatially and spectrally according to observational and instrumental conditions. The point-spread function used for spatial convolution of the 7090 line maps consists of a Gaussian core with $0^{\prime \prime} .25$ FWHM and Lorentzian wings (cf. Shelyag et al. 2004) with an amplitude chosen such that the RMS contrast of the continuum near to the line was degraded to $7 \%$ outside the sunspot. The spectral smearing was carried out by convolving with a Gaussian of $30 \mathrm{~m} \AA$ FWHM, corresponding to the spectral resolution of the TRIPPEL spectrograph at the Swedish Solar Telescope. The spatial and spectral resolutions of the degraded map of intensity profiles for the 7090 line are therefore comparable to data used by Bellot Rubio et al. (2010). For degrading the map of the CI 5380.34 $\AA$ line we used parameters corresponding to the CRISP 2D spectropolarimeter at the SST: a Gaussian with $0^{\prime \prime} .14$ FWHM combined with a Lorentzian chosen to yield 10\% RMS continuum contrast outside the sunspot for the spatial convolution and a Gaussian with a FWHM of $40 \mathrm{~m} \AA$ for the convolution in wavelength. All degraded images have been resampled to (spatial and spectral) pixel sizes equal to half of the FWHM of the corresponding Gaussian used for the convolution.

Line-of-sight (LOS) Doppler velocities are computed by means of the line bisector shifts for (relative) line intensity levels between $0 \%$ for the line core and $100 \%$ for the continuum level, in steps of $10 \%$. Shifts for intermediate intensity levels are determined by linear interpolation. Following standard observational procedure, we use the darkest part of sunspot as zero velocity reference. According to the varying formation height of the different parts of the line profile, bisectors near the continuum sample the velocities at deeper layers than those near the line core.

It should be kept in mind that the LOS speeds in our degraded maps still have to be considered as upper limits: we have assumed an ideal telescope and spectrograph, no noise and other instrumental effects. These can be quite important (cf. Joshi et al. 2011, see their Fig. 4 and Scharmer et al. 2011, see their Figs. S13-16).

Most of the results presented in the following section are for synthetic line profiles calculated along vertical lines of sight, corresponding to observations exactly at disk center. The effect of a non-zero viewing angle, i.e., inclined lines of sight, is considered in Sec. 3.3 .

\section{RESULTS}

\subsection{Velocities from synthetic line profiles}

The upper left panel of Figure 1 shows a synthetic continuum image for the $7090 \AA$ line at the original MHD simulation grid resolution of $32 \mathrm{~km}$. The granulation in the upper left part of the image has an rms contrast of about $15 \%$. White rectangles indicate the penumbral filaments that are considered in more detail in Sec. 3.2 below. The degraded and resampled continuum image corresponding to a spatial resolution of $0^{\prime \prime} .25$ resolution and $7 \%$ rms contrast is shown in the upper right panel of Figure 1 LOS velocity maps determined from the bisectors at $90 \%$ intensity level are shown in the lower part of the figure: the map on the left panel corresponds to the line profiles at original resolution while the map on the right panel is based on the (spatially and spectrally) degraded and resampled profiles. Note that bisectors at $90 \%$ intensity levels can be strongly affected in observational data by line blends and noise. In our line synthesis none of these effects is present. Therefore, our results give an optimistic estimate for the detectability of velocities based on line bisectors. All vertical (LOS) velocities are significantly lower after degradation. bf In particular, the downflow velocities at the edges of the filaments are reduced from $\sim 1000 \mathrm{~m} \mathrm{~s}^{-1}$ to typically less than $\sim 200 \mathrm{~m} \mathrm{~s}^{-1}$, so that they become much less visible in the degraded map. Only at a few locations the downflow speed reaches peak values of $\sim 300 \mathrm{~m} \mathrm{~s}^{-1}$. On the other hand, strong downflow patches at the outer peripery of the penumbra remain clearly detectable (e.g., Schlichenmaier \& Schmidt 1999, Franz \& Schlichenmaier 2009). These patches correspond to downturning Evershed flow in filaments that reenter the subsurface layers.

Figure 2 shows the corresponding results for the 5380 $\AA$ line using the $90 \%$ bisector. The continuum contrast is reduced from $24 \%$ at original resolution (upper left panel) to $10 \%$ in the degraded and resampled image (upper right panel) corresponding to $0^{\prime \prime} .14$ resolution. In comparison to the result for the $7090 \AA$ line, the reduction of the LOS velocities in the degraded maps is less severe: downflows at the edges of the penumbral filaments are reduced from $\sim 2000 \mathrm{~m} \mathrm{~s}^{-1}$ to $\mathrm{m} / \mathrm{s}$ only to $\sim 800 \mathrm{~m} \mathrm{~s}^{-1}$. Since the flow velocities in the simulated penumbral filaments are highest around optical depth unity (Rempel et al. 2009ab, Rempel 2011), these flows are better sampled by the $5380 \AA$ line, owing to its lower formation height.

Figure 3 shows LOS velocities along (from right to left) the artificial 'spectrograph slit' indicated by the white horizontal line in the upper right panel of Figure 1 The two upper panels illustrate, at original simulation resolution, how well the depth-dependent velocities are sampled by the two spectral lines. As the vertical flow speeds increase with depth in the atmosphere, the bisector shifts of the line wings (at $50 \%$ and $90 \%$ relative intensity) show higher LOS speeds than the line core shifts. Flow speeds determined from the deeper originating $5380 \AA$ line are generally higher. The lower two panels of Figure 3 illustrate the strong effect of spatial and 

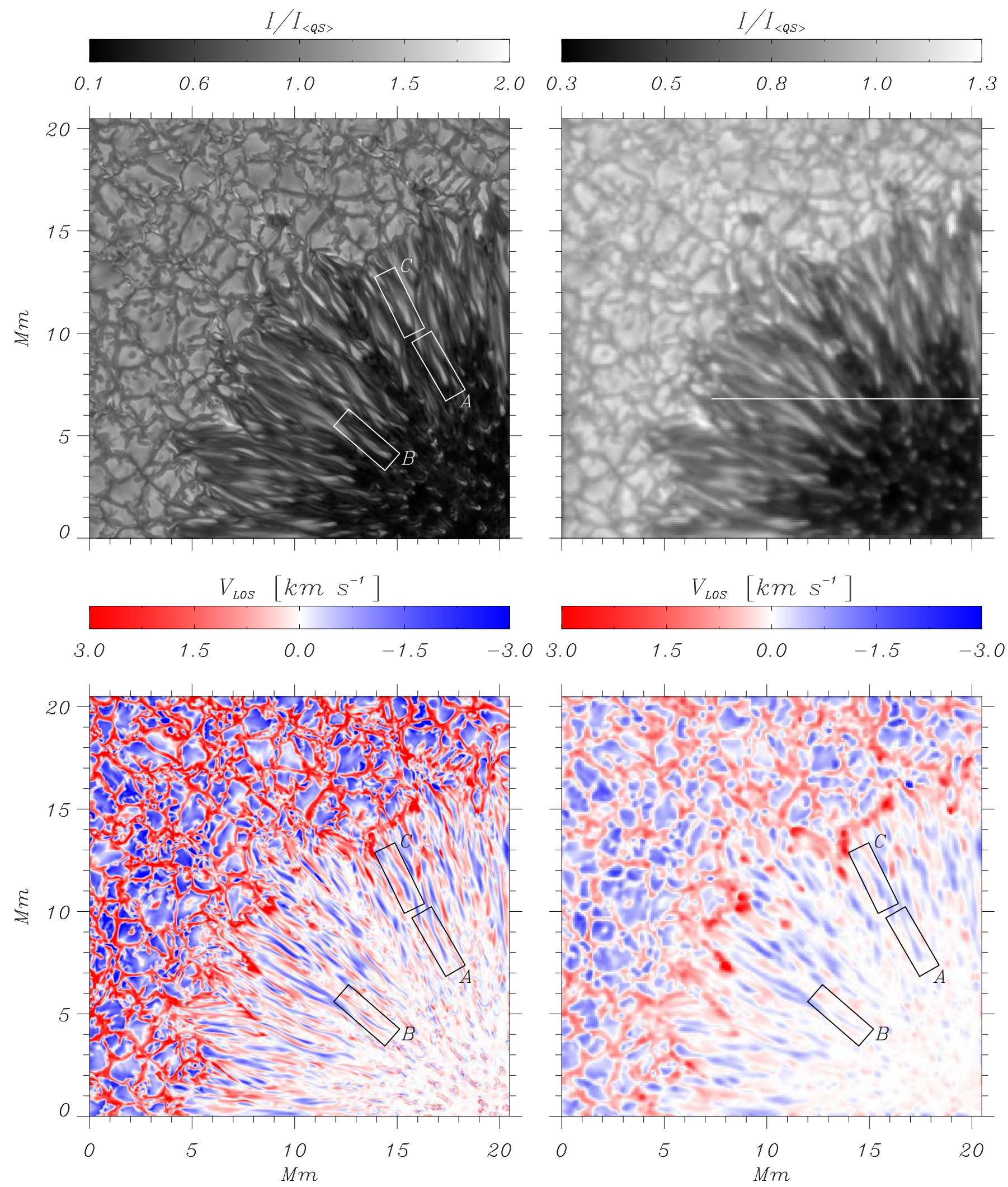

FIG. 1.- Upper row: synthetic continuum intensity map for the FeI $7090 \AA$ line at original simulation resolution (left) and at degraded resolution after convolution with a Gaussian profile of $0^{\prime \prime} .25$ FWHM (right). White rectangles indicate selected filaments that are studied in more detail in Figures 516 The horizontal line in the degraded image indicates the location of the artificial 'slit' for the velocity profiles shown in Figure 3 Lower row: bisector velocity at $90 \%$ relative intensity for the FeI $7090 \AA$ line at original simulation resolution (left) and at degraded resolution (right). The degraded images have been resampled to pixel size equal to half of the smearing FWHM. 

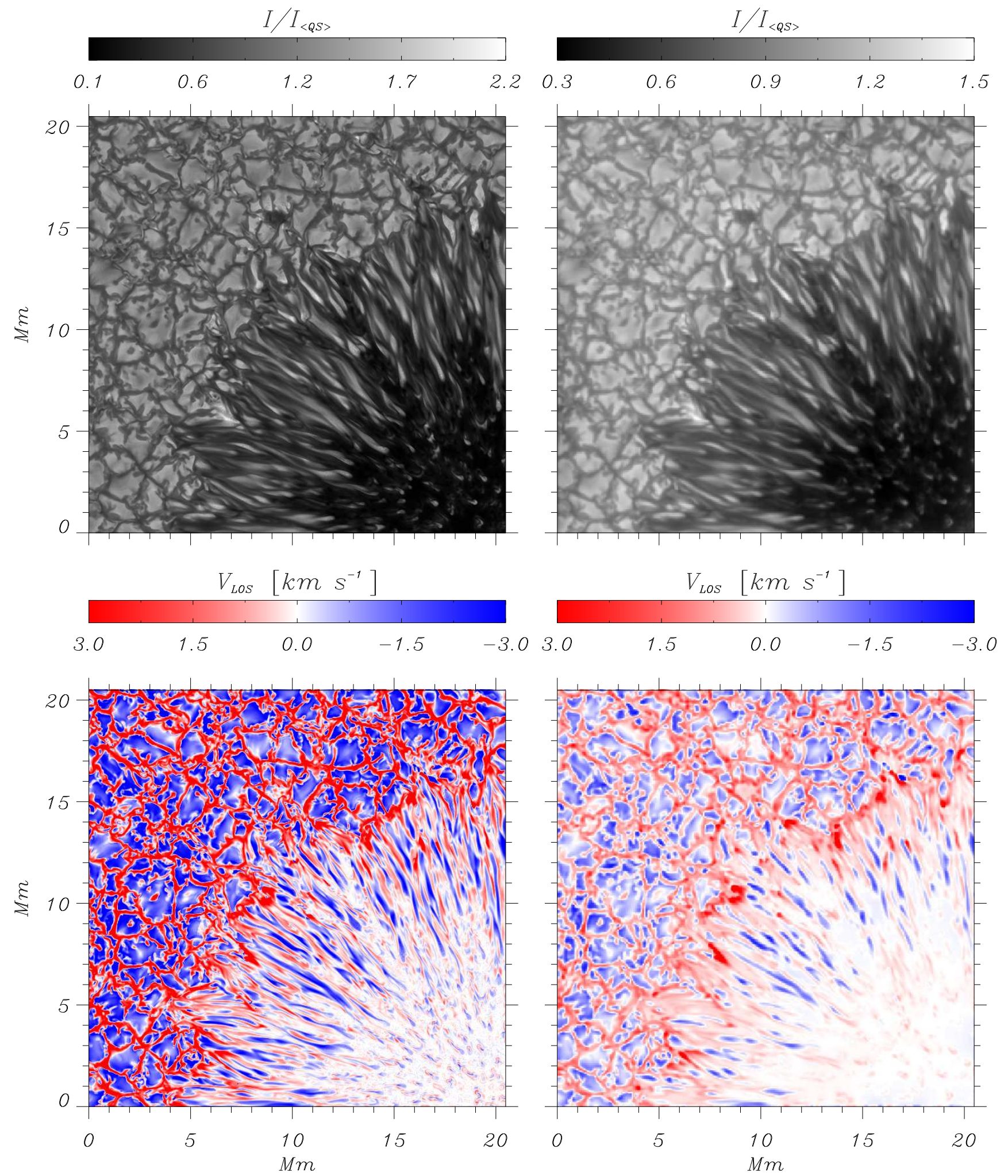

FIG. 2.- Same as Figure 1 but for the line CI 5380 A. The degraded images have been spatially convolved with a Gaussian of $0^{\prime \prime} .14$ FWHM. 

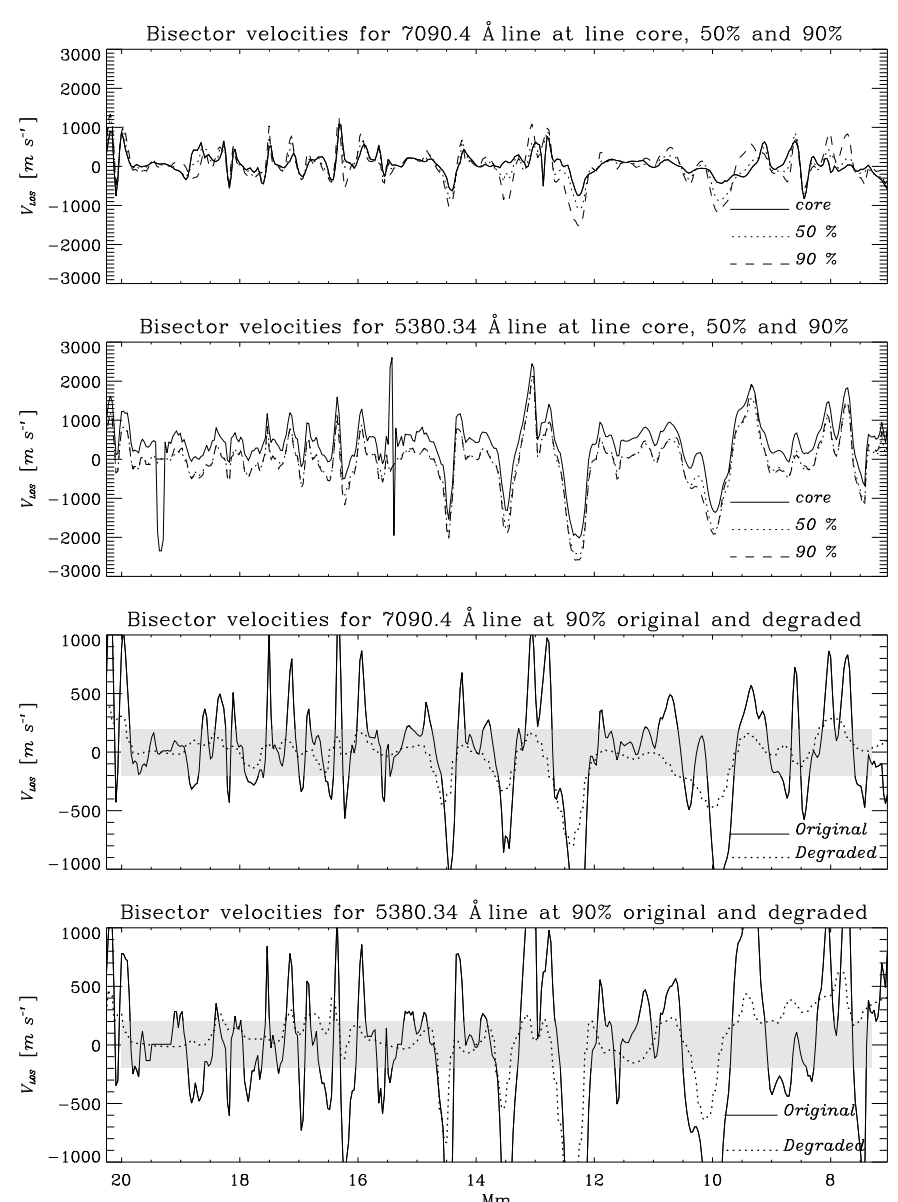

FIG. 3.- Bisector velocities along the horizontal 'slit' indicated in the upper left panel of Figure 1 The gray stripes indicate the typical uncertainty of observational measurements $\left( \pm 200 \mathrm{~m} \mathrm{~s}^{-1}\right)$.

spectral smearing on the flow speeds obtained from the bisectors at $90 \%$ relative intensity. While the up- and downflows of the overturning filament convection are conspicuous at original resolution, the downflow speeds in the degraded maps rarely exceed a typical observational detection limit of $\pm 200 \mathrm{~m} \mathrm{~s}^{-1}$ (Bellot Rubio et al. (2010) stated a detection limit of $\sim 50 \mathrm{~m} \mathrm{~s}^{-1}$ for their investigation with the error in the position of the zero of the velocities about $\sim 150 \mathrm{~m} \mathrm{~s}^{-1}$ ). On the other hand, the strongest upflows remain detectable. Similar results are found for the slit positions at $y=10 \mathrm{Mm}$ and $y=12 \mathrm{Mm}$ (not shown here). Generally, the downflows are more affected by the image degradation than the upflows. This is probably due to the smaller spatial scales of the downflows and their preferred occurence in dark regions, so that they are more susceptible to spatial smearing and straylight. This has also been found by Joshi et al. (2011, see their Fig. 4) and Scharmer et al. (2011, see their Figs. S13-16).

\subsection{Line-of-sight velocities of individual filaments}

We have selected three well-developed penumbral filaments in the simulation snapshot for a more detailed study of their velocity structure. The filaments $\mathrm{A}, \mathrm{B}$, and $\mathrm{C}$ are indicated in the upper left panel of Figure 1 . The actual vertical velocities from the simulation in vertical cuts perpendicular to the axes of the three filaments are shown in Figure 4. The upflows in the filaments and the downflows at their edges are well visible. The overplotted iso- $\tau$ lines show that the maximum speeds are found at or below optical depth unity, so that they are best

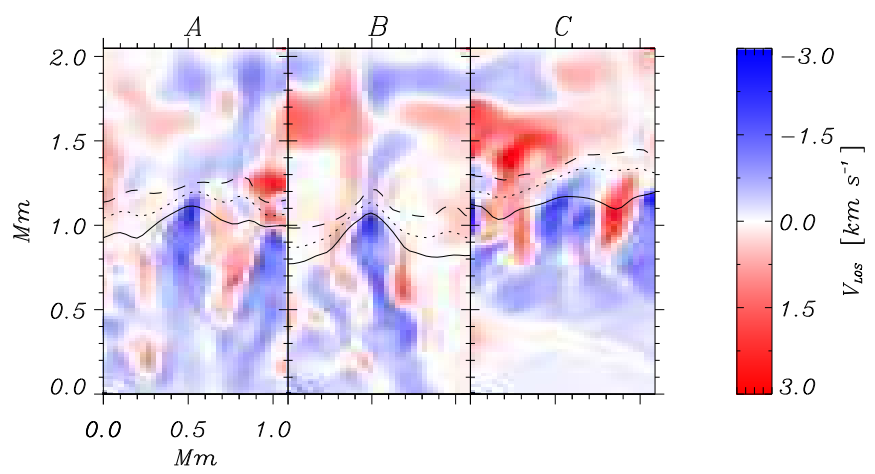

FIG. 4.- Vertical velocities in the simulation. Shown are vertical slices cut perpendicular to the direction of penumbral filaments $\mathrm{A}, \mathrm{B}$, and $\mathrm{C}$. The locations of the cuts are indicated on the intensity maps in Figures 5, 7 and 9 respectively. The lines indicate levels of constant optical depth for the continuum at $7090 \AA$ : $\tau=1$ (solid) $\tau=0.1$ (dotted), and $\tau=0.01$ (dashed).

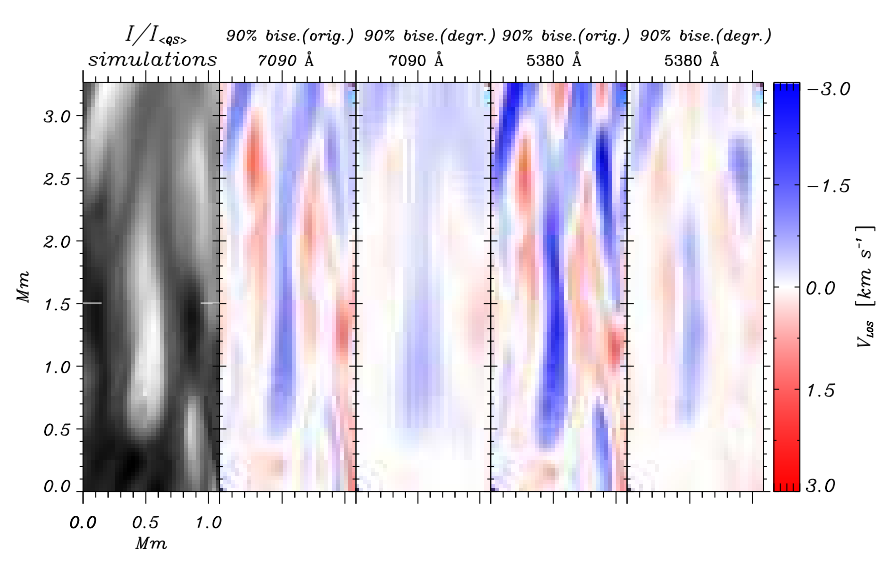

FIG. 5.- Intensity and velocity maps for filament A. The panels show, from left to right: intensity, bisector velocities for $90 \%$ relative intensity for FeI 7090 Å: original and degraded resolution, and bisector velocities at $90 \%$ relative intensity for CI 5380 Å: original and degraded resolution.

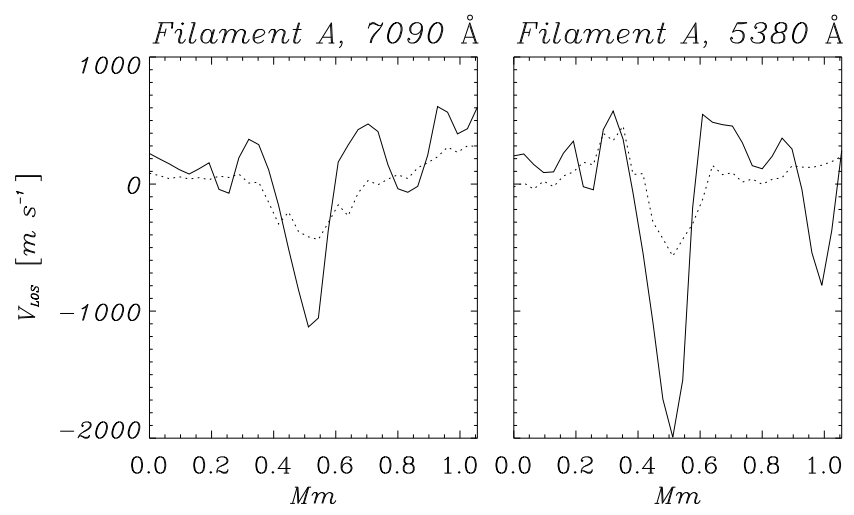

FIG. 6.- Velocity profiles along a horizontal cut perpendicular to filament A at $1.5 \mathrm{Mm}$, indicated on the intensity map in Figure 5 Shown are bisector velocities for $90 \%$ relative intensity at original resolution (solid lines) at and degraded resolution (dotted lines).

sampled by spectral lines with a low formation height.

The following figures show how the vertical flows in these filaments and their immediate surroundings are represented by the velocities determined from the line bisectors. Figure 5 shows maps of the continuum intensity and the $90 \%$ bisector velocities for the two lines and at both original and degraded resolution for filament $\mathrm{A}$. The velocity maps demon- 


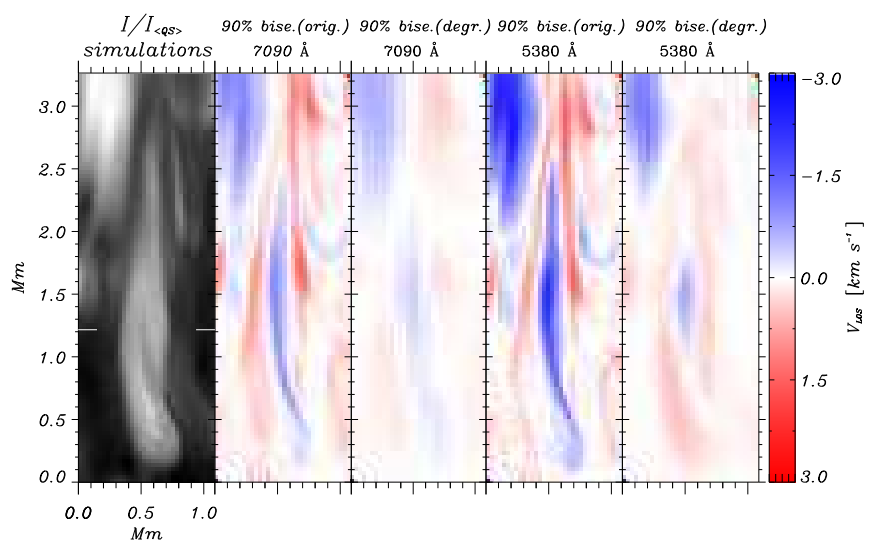

FIG. 7.- Same as Figure 5 but for filament B.

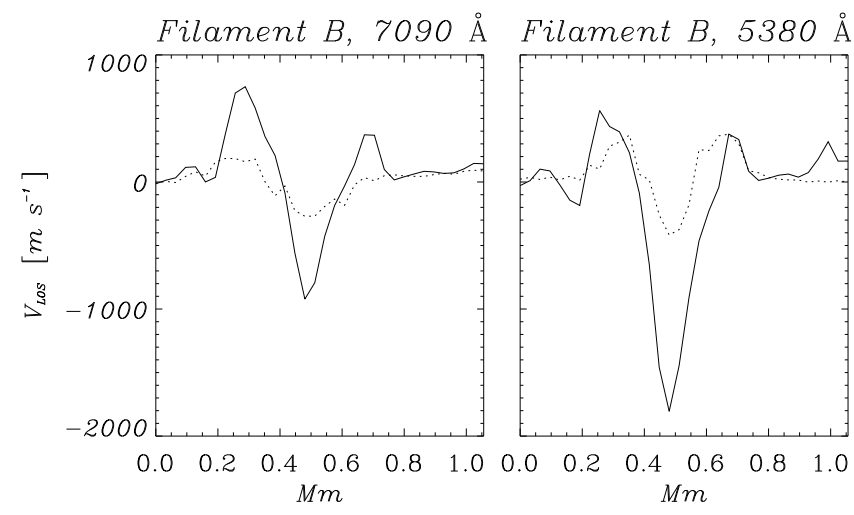

FIG. 8.- Same as Figure 6 but for filament B.

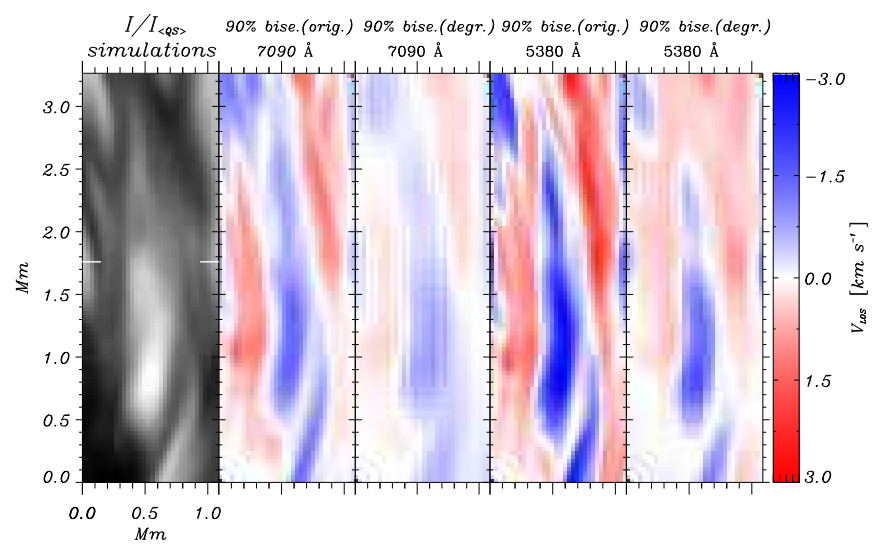

FIG. 9.- Same as Figure 5 but for filament C.

strate the strong effect of the degradation on the detectability of the vertical flows: while the typical velocity pattern with upflow in the filament and downflow along its edge is well represented at original resolution, it is difficult to see in the degraded velocity map from the $7090 \AA$ line. For the more deeply originating $5380 \AA$ line, a somewhat stronger velocity signal remains even for degraded resolution. This is made more quantitative in Figure 6 , which gives the bisector velocities along a horizontal 'slit' indicated by the two horizontal line segments in the continuum map in Figure 5. While the central upflow remains detectable even for the degraded map

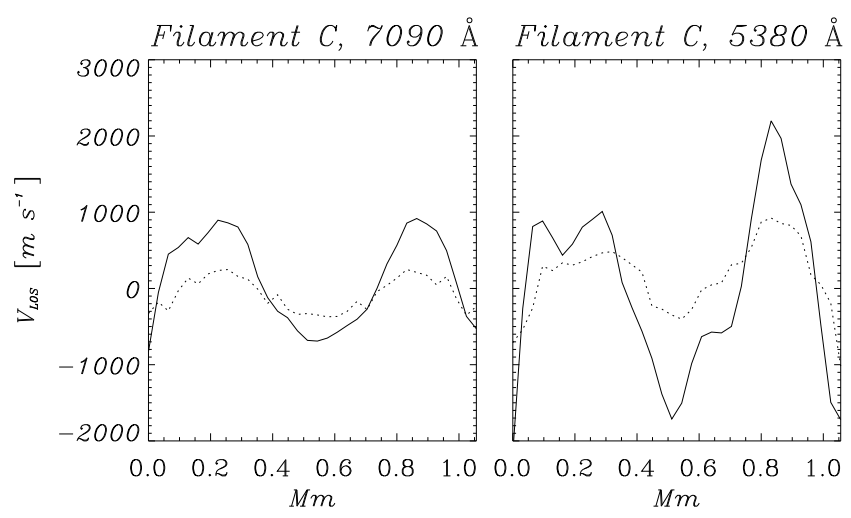

FIG. 10.- Same as Figure 6 but for filament C.

from the $7090 \AA$ bisector, the weaker downflows are reduced to values around or below the detection limit. Similar results are found for other cuts perpendicular to the filament. For the $5830 \AA$ line, the velocity signal is stronger and the downflow remains detectable for degraded resolution, at least at one side of the filament.

Qualitatively similar results are obtained for the other two filaments (Figures 7 7 10): the velocity signal is so strongly weakened by degradation for the $7090 \AA$ line that the downflow signal at the filament edges is reduced to an amplitude below the typical observational detectability limit of $\sim 200 \mathrm{~m} \mathrm{~s}^{-1}$ in most cases. For the $5380 \AA$ line, the situation is somewhat better: the deep origin of the line and the lower degree of spatial smearing (owing to the shorter wavelength) leave detectable velocity signals for both upflows and downflows, even after degradation.

\subsection{Effect of viewing angle}

When a sunspot is observed away from disk center, the observational detection of vertical velocities in penumbrae is complicated by the projection of the strong Evershed flow onto the direction of the LOS. This is relevant also for the results of Bellot Rubio et al. (2010), who studied a sunspot located only $5.4^{\circ}$ away from disk center. Since $\sin 5.4^{\circ} \simeq 0.094$, the component of a horizontal flow along the inclined LOS can reach a value of up to $\sim 10 \%$ of the horizontal flow speed, depending on the angle between the velocity vector and the direction toward disk center. With a horizontal Evershed flow of several $\mathrm{km} \mathrm{s}^{-1}$, this leads to projected LOS velocities of several hundred $\mathrm{ms}^{-1}$ for penumbral filaments directed towards (or away from) disk center. For the observations presented by Bellot Rubio et al. (2010) the projection of the Evershed flow leads to a blueshift, i.e., apparent upflows if (wrongly) interpreted as vertical flows. Their Figure 3 shows a clear trend toward increasing overall blueshift as the penumbral filaments become more aligned with the slit, which in turn is nearly parallel to the direction toward disk center. In fact, their bisectors at the $80-88 \%$ relative intensity level are all blueshifted, consistent with the maximum of the Evershed flow in the deeper layers.

To study the effect of the finite viewing angle on the bisector velocities from the simulated sunspot and to directly compare with the results of Bellot Rubio et al. (2010), we have redone the line synthesis assuming an inclination by $5.4^{\circ}$ to the vertical. Figure 11 shows the resulting spatially and spectrally degraded maps of the LOS velocity determined from the bisector shifts of the $7090 \AA$ line at $90 \%$ relative inten- 

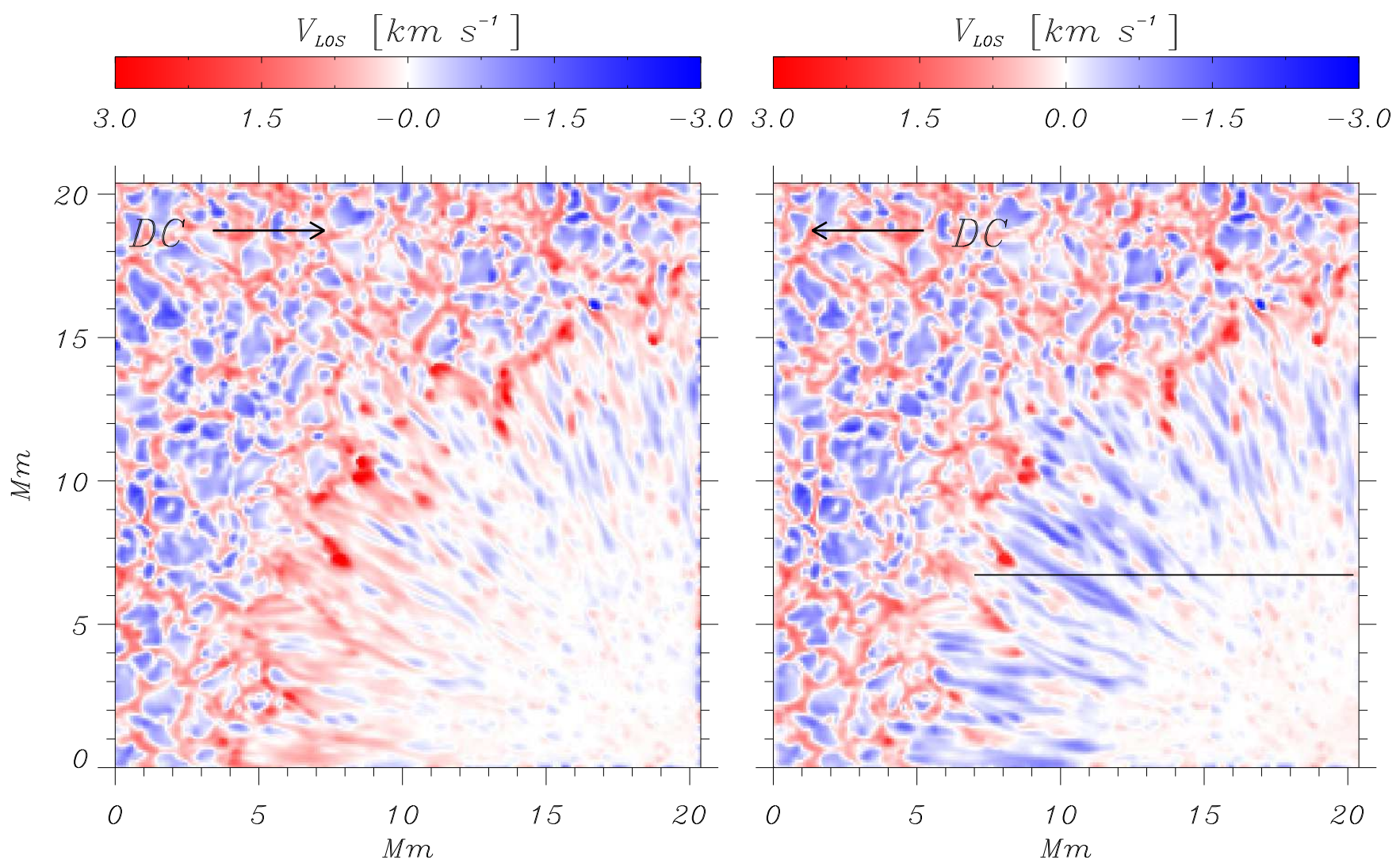

FIG. 11. - LOS velocity maps derived from the bisector shift at $90 \%$ relative intensity of FeI $7090 \AA$ for viewing angles inclined to the vertical by $5.4^{\circ}$. Left panel: disk center (DC) to the right; right panel: disk center to the left.

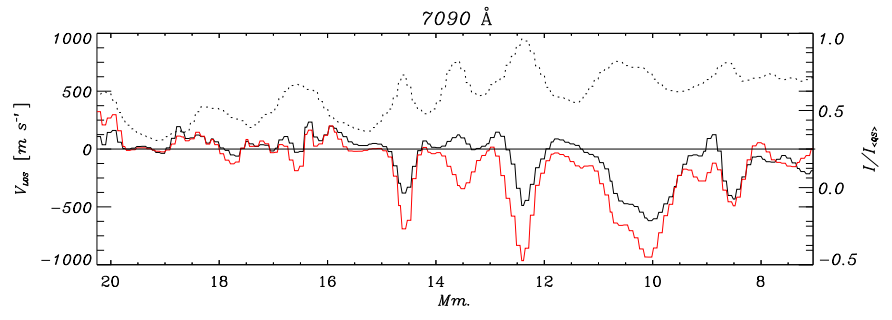

FIG. 12.- Profiles of the LOS velocity for a viewing angle of $5.4^{\circ}$ along the 'slit' indicated on the right panel of Figure 11 Note that we reversed the horizontal direction along the 'slit' compared to Figure11 in order to present quantities directly comparable to Figure 3 in Bellot Rubio et al. (2010). LOS velocities were derived from bisector shifts of FeI $7090 \AA$ A. The black solid line corresponds to the average bisectors for $0-8 \%$ relative intensity (line core) and the red line to average bisectors for $80-88 \%$ relative intensity (line wing). The dotted line shows the profile of the continuum intensity at 7090 $\AA$.

sity level. Disk center is located to the left or to the right, respectively, in the direction of the slit indicated in the right panel. In the latter case (right panel), which corresponds to the observations of Bellot Rubio et al. (2010), the penumbral region is clearly dominated by blueshifts due to the projection of the Evershed flow. LOS velocity profiles along the slit are shown in Figure 12, As the penumbral filaments become more aligned with the slit, the projection of the Evershed flow onto the direction of the LOS increases. In addition, the Evershed flow speed increases outward in the penumbra. The combination of these two effects leads to the downward tilt in the velocity profile shown in Figure 12, which is more conspicuous for the bisectors in the line wings since the Evershed flow is faster in the deeper layers. These properties of the ve- locity profiles are very similar to those found by Bellot Rubio et al. (2010, see their Figure 3), strongly suggesting that both result from the projection of the Evershed flow. The difference between the bisector shifts of line core and line wings is even higher in the observations, indicating that the increase of the Evershed flow with depth is possibly underestimated in the simulations.

\section{DISCUSSION AND CONCLUSION}

We have found that the observational detection of overturning convection in penumbral filaments can be severely compromised by a) finite spatial and spectral resolution and b) projection of the Evershed flow for observations not taken exactly at disk center. In particular, the relatively weak downflows along the edges of filaments suffer most from these effects: for the $7090 \AA$ line their observational signal is reduced to values below the limit of reliable detection around $\sim 200 \mathrm{~m} \mathrm{~s}^{-1}$ in most cases. The situation is somewhat better for the $5380 \AA$ line, which samples the higher velocity amplitudes in the deeper layers of the atmosphere owing to its lower formation height. Obviously, better spatial resolution also significantly improves the detectability of these smallscale velocity structures.

The combined effects of degradation and projection probably also affected the observations of Bellot Rubio et al. (2010), so that they cannot decide upon the presence or absence of overturning convection in the penumbra exceeding the stated detection limit of $\sim 50 \mathrm{~m} \mathrm{~s}^{-1}$. Note that our analysis did not take into account the effects of noise (due to the detector, electronics, and seeing) in the observed line profiles. Comparing our degraded images with Figure 2 of Bellot Ru- 
bio et al. (2010) also indicates that the spatial degradation of the observations is stronger than in our attempts to simulate them. However, the main problem of these observations is the projection of the Evershed flow onto the LOS. Even at a heliocentric angle of only $5.4^{\circ}$, up to $10 \%$ of the Evershed flow is projected onto the LOS and the trend in the velocity profile shown in Figure 3 of Bellot Rubio et al. (2010) clearly indicates that this effect is present in their observations. This is also supported by the fact that the bisector blueshifts are significantly stronger for the line wings than for the line core. Therefore, the absence of redshifted bisectors must not necessarily be taken as evidence for the absence of downflows since the projection of the Evershed effect easily leads to a blueshift of several $100 \mathrm{~m} \mathrm{~s}^{-1}$.

On the other hand, the non-detection of overturning penumbral convection by Franz \& Schlichenmaier (2009) and Bellot Rubio et al. (2010) could, in principle, indicate that these flows are overestimated in current numerical simulations owing to insufficient spatial resolution, artificial boundary conditions, and possibly insufficient domain depth. We have recently carried out a convergence study (Rempel, in preparation) for which the numerical grid spacing was varied between $96 \mathrm{~km}$ and $16 \mathrm{~km}$ in the horizontal directions and between $32 \mathrm{~km}$ and $12 \mathrm{~km}$ in the vertical direction. The simulation analyzed here is the second best resolved simulation of that series (the highest resolution case has not yet been computed with non-grey radiative transfer). Since we do not use explicit diffusivities, changes in the grid spacing directly affect the overall numerical dissipation (which scales at least linearly with grid spacing near discontinuities but with a higher order in well-resolved regions). The amount of overturning motions (characterized by the vertical rms velocity at $\tau=1$ ) turns out to be robust: it is directly tied to the penumbral brightness, which does not change significantly with resolution. On the other hand, we find that the average width of filaments decreases somewhat with increasing resolution since they are still only marginally resolved. It is therefore well conceivable that our current simulation overestimates the visibility of convective motions in the penumbra. We also investigated the influence of the top boundary condition. While the overall extent of the penumbra depends on the choice of the boundary condition, the detailed structure of the magnetoconvection is mostly unaffected. We find an approximate relationship of the form $I \propto \sqrt{{ }_{z}^{\text {rms }}(\tau=1)}$ between the azimuthally averaged bolometric intensity and the rms vertical velocity defined through the azimuthal average at each radial position of the spot (Rempel 2011), independent of the boundary condition and extent of the penumbra. As a consequence, the prediction that the vertical rms velocity in the penumbra should be about half of the value found in quiet sun is fairly robust; it is also consistent with the width of distribution functions inferred from Hinode data by Franz \& Schlichenmaier (2009).

Our analysis indicate that the $5380 \AA$ line is better suited than the $7090 \AA$ line for the detection of overturning convection in penumbral filaments. This is due to the fact that this line originates deeper in the atmosphere (and thus samples higher velocities) and that the shorter wavelength affords a higher spatial resolution for a given telescope. Even after degradation, many downflows exceed a typical detectability threshold of $200 \mathrm{~m} \mathrm{~s}^{-1}$. Results on penumbral up- and downflows using the $5380 \AA$ line were obtained by Schlichenmaier \& Schmidt (1999) with the German VTT on Tenerife and recently by Joshi et al. (2011) and Scharmer et al. (2011) with the CRISP 2D spectropolarimeter at the SST. In any case, such observations are complicated by the projection of the strong Evershed flows and therefore should be carried as near as possible to disk center - assuming that the Evershed flow is exactly horizontal, which obviously will not always be the case.

Discussions with Drs. J. Hirzberger and A. Lagg are gratefully acknowledged. The authors thank Dr. B. Lites for helpful comments on the manuscript. LB is grateful to InterUniversity Centre for Astronomy and Astrophysics (IUCAA) Reference Center at Department of Physics, Mohanlal Sukhadia University, Udaipur, India, for providing computational facilities during his visit.

The National Center for Atmospheric Research (NCAR) is sponsored by the National Science Foundation. Computing resources for the sunspot model utilized in this investigation were provided through NCAR's Computational Information Systems Laboratory (CISL) as well as through the NSF Teragrid at the National Institute for Computational Sciences (NICS) under grant TG-AST100005.

\section{REFERENCES}

Berdyugina, S. V.; Solanki, S. K.; Frutiger, C., 2003, A\&A, 412, 513 Bellot Rubio, L.R., Schlichenmaier, R. Langhans, K., 2010, ApJ, 725, 11

Bharti, L., Joshi, C. and Jaaffrey, S. N. A.: 2007a, ApJ, 669, L57

Bharti, L., Jain, R. and Jaaffrey, S. N. A.: 2007b, ApJ, 665, L79

Bharti, L., Rimmele, T., Jain, R., Jaaffrey, S. N. A., Smartt, R. N.: 2007c, MNRAS, 376, 1291

Bharti, L.; Joshi, C.; Jaaffrey, S. N. A.; Jain, R.: 2009, MNRAS, 393, 65

Bharti, L.; Beek, B.; Schüssler, M.: 2010a, A\&A, 510A, 12B

Bharti, L., Solanki, S. K., \& Hirzberger, J. 2010b, ApJ, 722, 194

Fleck, B., 1991, Ph.D. thesis, Univ. Würzburg, (1991)

Franz, M., Schlichenmaier, R. 2009, A\&A, 508, 1453

Heinemann, T., Nordlund, Å., Scharmer, G. B., \& Spruit, H. C. 2007, ApJ, 669, 1390

Ichimoto, K., et al. 2007, Science, 318, 1597

Joshi, Jayant, Pietarila, A., Hirzberger, J., Solanki, S. K., Aznar Cuadrado, R. and Merenda, L. 2011, ApJ, 734, L18

Katsukawa, Y., Jurčák, J., 2010, A\&A, 524, A20

Louis, Rohan E.; Bellot Rubio, Luis R.; Mathew, Shibu K.; Venkatakrishnan, P., 2011, ApJ, 727, 49

Márquez, I., Sánchez Almeida, J. \& Bonet, J. A: 2006, ApJ, 638, 553

Ortiz, A., Bellot Rubio, L. R., Rouppe van der Voort, L., 2010, ApJ, 713, 1282

Rempel, M., Schüssler, M., \& Knölker, M. 2009a, ApJ, 691, 640

Rempel, M., Schüssler, M., Cameron, R. H., \& Knölker, M. 2009b, Science, 325,171
Rempel, M. 2010, Proc. of IAU Symp. 273, arXiv:1011.0981

Rempel, M. 2011, ApJ, 729, 5

Rimmele, T. 2008, ApJ, 672, 684

Rouppe van der Voort, L. H. M. Bellot Rubio, L. R. Ortiz, A. 2010, ApJ, 718, 78

Sánchez Almeida, J., Márquez, I., Bonet, J. A., \& Domínguez Cerdeña, I. 2007, ApJ, 658, 1357

Scharmer, G. B., Bjelksjo, K., Korhonen, T. K., Lindberg, B., \& Petterson, B. 2003, Proc. SPIE, 4853, 341

Scharmer, G. B., Nordlund, Å., \& Heinemann, T. 2008, ApJ, 677, L149

Scharmer, G. B., Henriques, V. M. J., Kiselman, D,\& de la Cruz Rodríguez, J. 2011, Science, 10.1126/science.1206429

Schlichenmaier, R., Schmidt, W., 1999, A\&A, 349, L37

Schüssler, M., Vögler, A., 2006, ApJ, 541, L73

Shelyag, S., Schüssler, M., Solanki, S. K., Berdyugina, S. V., Vögler, A., 2004, A\&A, 427, 335

Vögler, A., Shelyag, S. Schüssler, M., Cattaneo, F., Emonet, T. Linde, T., 2005, A\&A, 429, 335

Zakharov, V., Hirzberger, J., Riethmüller, T. L., Solanki, S. K., \& Kobel, P. 2008, A\&A, 488, L17 